\title{
Changes of pediatric respiratory tract infections in Chongqing Health Center for Women and Children during COVID-19
}

\author{
Xu Xiaoxiao ${ }^{1}, \mathrm{Xu} \mathrm{Mingdeng}{ }^{2}$, and Li Xuemei ${ }^{1}$ \\ ${ }^{1}$ Chongqing Health Center for Women and Children \\ ${ }^{2}$ The Third Affiliated Hospital of Chongqing Medical University
}

September 16, 2020

\begin{abstract}
By comparing the outpatient volume of pediatric patients and the outpatient volume of respiratory tract infections in Chongqing Maternal and Child Health Care Hospital from 2017 to June 2020, the changes in the outpatient volume of pediatric patients and the outpatient volume of respiratory tract infections during COVID-19 were summarized and analyzed, so as to provide basis for disease prevention.
\end{abstract}

\section{Hosted file}

COVID-19 \begin } \{ \text { CJK\} \{UTF8\} \{gbsn\}. \end \{CJK\}\selectlanguage \{english\}docx available } https://authorea.com/users/359584/articles/481465-changes-of-pediatric-respiratorytract-infections-in-chongqing-health-center-for-women-and-children-during-covid-19 